\title{
PREVALENCE OF ASTHMA, RHINITIS ALLERGIC AND ECZEMA AMONG MIDDLE SCHOOL STUDENTS IN TABRIZ (NORTHWESTERN OF IRAN)
}

\author{
M. Sadeghi Shabestari ${ }^{1}$, L. Sahebi ${ }^{2}$ \\ ${ }^{1}$ Immunology Allergy, Children's Hospital ,TB and Lung Research Center, Tabriz University, ${ }^{2}$ TB and \\ Research Center, Tabriz University, Tabriz, Iran
}

Background: Asthma, rhinitis allergic, and eczema have an extensive epidemiologic diversity in various geographic areas. This study was performed on middle school students for investigation of the prevalence of atopic syndrome and their risk factors.

Methods: This was a cross-sectional study that was performed on 1,508 students in the city of Tabriz (Northwestern Turkish-populated region of Iran). Data collection tool was the International Study of Asthma and Allergies in Childhood questionnaire.

Results: Cumulative and Periodic wheezing and diagnosed asthma prevalence were 3.7\%, 2.9\%, and 2\% respectively. Cumulative and Periodic Rhinitis Allergic and Hay Fever Diagnosed prevalence were 17.1\%, $16.0 \%$, and $13.6 \%$ respectively. Cumulative and Periodic Nocturnal Rush and Eczema Diagnosed prevalence were $5.4 \%, 4.7 \%$, and $7.3 \%$ respectively. Asthma symptoms were more prevalent in the western part of the city than in the central and eastern parts, Boys were more likely to have symptoms of rhinitis and eczema than girls.

Conclusion: The small difference observed in this study between Cumulative and Periodic Rhinitis Allergic prevalence could be caused by the recent sharp increase in rhinitis allergic prevalence which is increasing rapidly. In regard to the factors contributing to the higher prevalence of asthma in the western part of the city in comparison with the central and eastern parts in relation to demographic, environmental, and even ethnic variables, conduction of an ecological survey in this geographical region and even in adjacent countries could be helpful. 